\title{
SURFACE PHOTOVOLTAGE IN PHOTOEMISSION STUDIES AT Si/InP(110) HETEROJUNCTIONS*
}

\author{
B. ADAMOWICZ \\ Institute of Physics, Silesian Technical University \\ Krzywoustego 2, 44-100 Gliwice, Poland \\ C. Ottaviani, C. Quaresima and P. Perfetti
}

Istituto di Struttura della Materia/CNR, Via E. Fermi 38, 00044 Frascati, Italy

We discuss the surface photovoltage effect observed in photoemission experiment performed at room $(300 \mathrm{~K})$ and low $(120 \mathrm{~K})$ temperatures on $\mathrm{Si} / \mathrm{InP}(110)$ heterojunctions for a thin Si coverage on $n$ - and $p$-doped InP substrates. The theoretical analysis of the surface photovoltage effect has been performed on the basis of thermionic and thermionic-field emission models of transport processes in Schottky barriers.

PACS numbers: $73.20 .-r, 73.20$.At

\section{Introduction}

The surface photovoltage phenomenon, i.e., a change in band bending induced by photons at semiconductor surfaces and interfaces with built-in potential barriers, has recently been observed in UPS experiments at many metal-semiconductor Schottky junctions [1] and only in a few cases of semiconductor-semiconductor interfaces $[2,3]$.

In Ref. [3] dell'Orto et al. have reported on the surface photovoltage at the a:Si $/ n-\operatorname{InP}(110)$ heterojunction at $120 \mathrm{~K}$, generated by ultraviolet synchrotron radiation in photoemission studies. It is interesting that for the junction with a $p$-InP substrate they did not observe any surface photovoltage effect (SPV). They described the SPV mechanism qualitatively in terms of generation, transport, and recombination of excess electron-hole pairs at the heterojunction. In this work we discuss those results on the basis of both the thermionic and thermionic-field emission models of transport phenomena in Schottky barriers $[4,5]$.

"One of us (B.A.) undertook this work with the support of the ICTP Programme for Training and Research in Italian Laboratories, Triest (Italy) and partially within the Silesian Technical University BK/RMF-1/95 Research Program. 


\section{2. $\mathrm{Si} / n$-InP(110) heterojunction}

The $\mathrm{Si} / n$ - $\operatorname{InP}(110)$ heterojunction was obtained by evaporation of $1 \mathrm{~nm}$ of a:Si on the $\operatorname{InP}(110)$ surface cleaved under UHV conditions $\left(5 \times 10^{-10} \mathrm{mbar}\right)$ and studied in situ by means of photoelectron spectroscopy at 300 and $120 \mathrm{~K}$ [3]. A pronounced shift of $0.3 \mathrm{eV}$ towards higher binding energies was observed in the UPS spectra measured at $120 \mathrm{~K}$ compared to the spectra at $300 \mathrm{~K}$. This result was attributed to the non-equilibrium state (SPV) induced by the ultraviolet synchrotron radiation at the interface. Figure 1a illustrates this situation. Photoelectrons generated by UV radiation are driven away from the interface by the potential of the depletion layer, whereas photoholes go into the opposite direction.
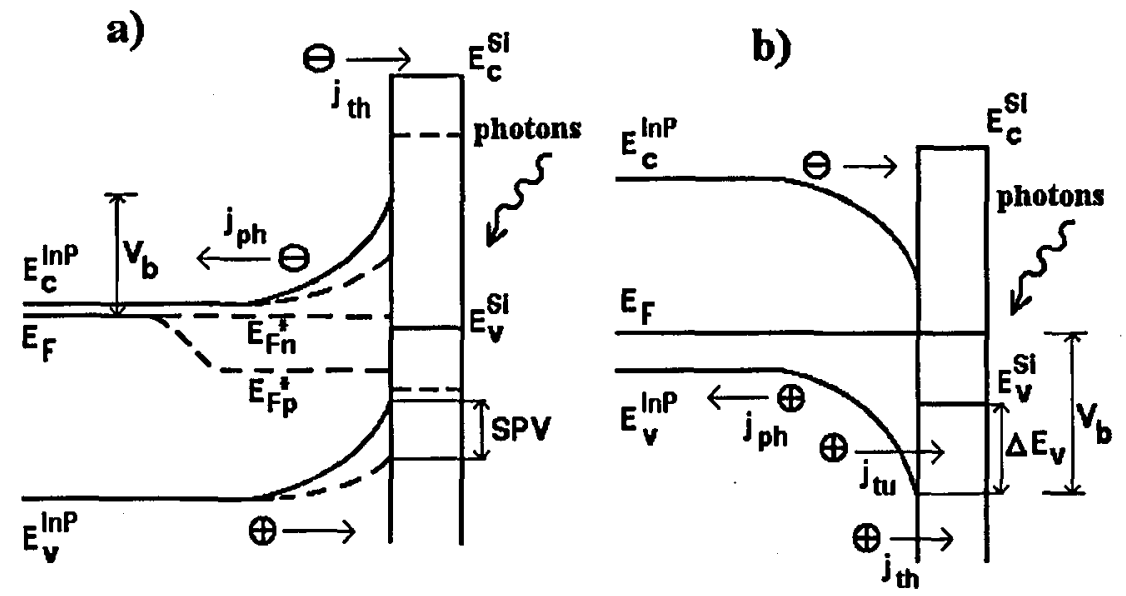

Fig. 1. Band structure of $\mathrm{Si} / n$-InP (a) and $\mathrm{Si} / p$-InP interfaces (b) in the dark (solid lines) and under illumination (dashed lines). In the case (b), SPV $=0$, as in the experiment. The valence band discontinuity $\Delta E_{\mathrm{v}}=0.6 \mathrm{eV}$ [3]. The arrows point the excess carrier flows.

This photocurrent $\left(j_{\mathrm{ph}}\right)$ is restored mainly by the thermionic emission current of electrons from $\mathrm{InP}$ to $\mathrm{Si}\left(j_{\mathrm{th}}\right)$ and then recombination at the interface. Another possible restoring process, the tunneling of electrons through the interface barrier from $n$-InP to $\mathrm{Si}$, seems to be less probable because of the much lower density of electron states near the midgap of $\mathrm{Si}$, where the tunneling can occur, than near the edges of the gap (U-shape distribution of the interface states). As a result, a partial flattening of the energy bands (SPV) appears. The surface photovoltage can also be measured in an UPS experiment as a shift in the Fermi level at the interface, i.e., a distance between the Fermi level in the dark $\left(E_{\mathrm{F}}\right)$ and the hole quasi-Fermi level $\left(E_{\mathrm{Fp}}^{*}\right)$ under illumination. The state of the shined heterojunction is similar to the forward-biased Schottky diode. Therefore, according to the thermionic emission theory, the photocurrent $j_{\mathrm{ph}}$ is given by the following formula [6]:

$$
j_{\mathrm{ph}}=A^{*} T^{2} \exp \left(-q V_{\mathrm{b}} / k T\right)\left[\exp \left(q V_{\mathrm{ph}} / k T\right)-1\right],
$$


where $A^{*}=4 \pi m_{n} q k^{2} h^{-3}$ is the Richardson constant of InP, $m_{n}$ is the electron effective mass (for InP $m_{n}=0.077 m_{0}$ [7], where $m_{0}$ is the free electron mass), $k$ is the Boltzmann constant, $h$ is the Planck constant, $T$ is the temperature, $q$ is the electron charge, $V_{\mathrm{b}}$ is a distance between $E_{\mathrm{c}}$ and $E_{\mathrm{F}}$ at the interface and $V_{\mathrm{ph}}$ is the surface photovoltage.

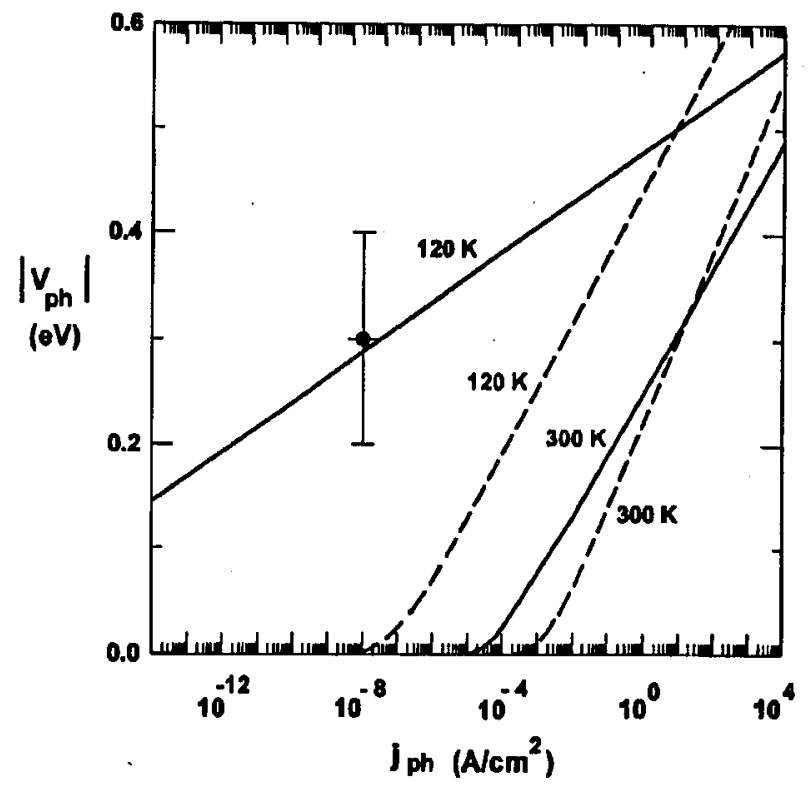

Fig. 2. Theoretical dependences of the absolute value of SPV vs. photocurrent density for $\mathrm{Si} / n$-InP (solid lines) and $\mathrm{Si} / p$-InP (dashed lines). The black circle represents experimental values of SPV and $j_{\mathrm{ph}}$ at $120 \mathrm{~K}$ for $\mathrm{Si} / n$-InP; for $\mathrm{Si} / p-\mathrm{InP}, \mathrm{SPV}=0$.

The results of calculations of $V_{\mathrm{ph}}$ vs. $j_{\mathrm{ph}}$ at 120 and $300 \mathrm{~K}$, on the basis of Eq. (1), and for $V_{\mathrm{b}}=0.6 \mathrm{eV}$. [8] are presented in Fig. 2 (solid lines). It is clear that the experimental values of $V_{\mathrm{ph}}(0.3 \mathrm{eV})$ and $j_{\mathrm{ph}}$ (estimated at about $10^{-8} \mathrm{~A} / \mathrm{cm}^{2}$ ), represented by the black circle in Fig. 2, are in good agreement with the theoretical curve. Moreover, we see the theoretical value of SPV at $300 \mathrm{~K}$ is about zero for $j_{\mathrm{ph}}=10^{-8} \mathrm{~A} / \mathrm{cm}^{2}$.

\section{3. $\mathrm{Si} / p$-InP(110) heterojunction}

For the a:Si $/ p$-InP heterojunction, contrary to the previous system, there was no shift in the UPS spectra (no SPV) at $120 \mathrm{~K}$ [3]. Figure $1 \mathrm{~b}$ shows the band scheme of this heterojunction. The UV photons generate excess electrons and holes which are driven into opposite directions $\left(j_{\mathrm{ph}}\right)$. The restoring current consists of both the thermionic $\left(j_{\text {th }}\right)$ and tunneling $\left(j_{\text {tu }}\right)$ currents through the "notch" in the valence band. Therefore, the theoretical analysis of SPV was carried out on the basis of the thermionic-field emission theory [6]. The photocurrent $j_{\mathrm{ph}}$ is given by the formula

$$
j_{\mathrm{ph}}=j_{\mathrm{th}}+j_{\mathrm{tu}}=j_{\mathrm{s}} \exp \left(V_{\mathrm{ph}} / E_{0}\right)\left[1-\exp \left(-q V_{\mathrm{ph}} / k T\right)\right],
$$


where

$$
\begin{aligned}
& E_{0}=E_{00} \operatorname{coth}\left(q E_{00} / k T\right) \text { and } E_{00}=h /(4 \pi)\left[N_{\mathrm{d}} /\left(m_{p} \varepsilon \varepsilon_{0}\right)\right]^{0.5}, \\
& j_{\mathrm{s}}=j_{\mathrm{m}} q \frac{\left[\pi E_{00}\left(V_{\mathrm{b}}-V_{\mathrm{ph}}-V_{n}\right)\right]^{0.5}}{\left[k T \cosh \left(q E_{00} / k T\right)\right]} \exp \left[-\left(V_{\mathrm{b}}-V_{n}\right) / E_{0}\right]
\end{aligned}
$$

and

$$
j_{\mathrm{m}}=A^{*} T^{2} \exp \left(-q V_{n} / k T\right) .
$$

In Eqs. (2)-(5) $m_{p}$ is the hole effective mass, $\varepsilon$ and $\varepsilon_{0}$ are the semiconductor and vacuum permittivities (for InP $m_{p} / m_{0}=0.64$ and $\varepsilon=12.61[7]$ ), $V_{\mathrm{b}}$ and $V_{n}$ are the separations between $E_{F}$ and $E_{\mathrm{v}}$ at the interface and in the bulk of InP, respectively, and $N_{\mathrm{d}}$ is the acceptor concentration $\left(N_{\mathrm{d}}=1.6 \times 10^{19} \mathrm{~cm}^{-3}\right)$. Using the relations given above and assuming $V_{\mathrm{b}}=0.8 \mathrm{eV}$ [8], we calculated the dependences of $V_{\mathrm{ph}}$ vs. $j_{\mathrm{ph}}$ (dashed curves in Fig. 2). It is clear that at both 300 and $120 \mathrm{~K}$, for $j_{\mathrm{ph}}=10^{-8} \mathrm{~A} / \mathrm{cm}^{2}$, the value of SPV is about zero, as in the experiment.

The analysis presented indicates the differences in the restoring process of photon-induced charges at $\mathrm{Si} / \operatorname{InP}(110)$ heterojunctions with $n$ - and $p$-doped substrates. In the case of $n$-InP, the thermionic current of excess electrons from InP to Si prevails whereas in the case of $p$-InP the tunneling of holes through the potential "notch" is crucial. A more detailed analysis of these processes, including recombination at the interface, we will discuss in a future paper.

\section{References}

[1] K. Horn, M. Alonso, R. Cimino, Appl. Surf. Sci. 56-58, 271 (1992).

[2] Xiaohua Yu, A. Raisanen, G. Haugstad, G. Ceccone, N. Troullier, A. Franciosi, Phys. Rev. B 42, 1872 (1990).

[3] T. dell'Orto, G. De Stasio, M. Capozi, C. Ottaviani, C. Quaresima, P. Perfetti, Y. Hwu, G. Margaritondo, Appl. Surf. Sci. 65/66, 789 (1993).

[4] Heterojunction Band Discontinuities, Eds. F. Capasso, G. Margaritondo, North-Holland, Amsterdam 1987.

[5] M.A. Herman, Semiconductor Heterojunctions, PWN, Warsaw 1987 (in Polish).

[6] E.H. Rhoderick, R.H. Williams, Metal-Semiconductor Contacts, Clarendon, Oxford 1988.

[7] Landolt-Bornstein Numerical Data and Functional Relationships in Science and Technology, Ed. O. Madelung, New Series, Vol. 17a, Springer, Berlin 1982.

[8] A.D. Katnani, G. Margaritondo, Phys. Rev. B 28, 1944 (1983). 